\title{
Österreichischer Forschungs- und Technologiebericht 2020 FACTSHEET
}

\section{Aktuelle Entwicklungen in Österreich}

\section{F\&E-Ausgaben}

Laut revidierter Globalschätzung der Statistik Austria betrugen die Ausgaben für Forschung und experimentelle Entwicklung (F\&E) im Jahr 2019 12,69 Mrd. $€$ und lagen damit um 4,8\% über dem Wert von 2018 (12,11 Mrd. €).

Die geschätzte F\&E-Quote (Anteil der Bruttoinlandsausgaben für Forschung und Entwicklung gemessen am Bruttoinlandsprodukt) betrug 2019 $3,18 \%$, was einen leichten Anstieg im Vergleich zum Vorjahr (2018: 3,14 \%) bedeutet. Österreich liegt damit bereits zum sechsten Mal in Folge über dem europäischen Zielwert von $3 \%$.

\section{Finanzierung nach Sektoren}

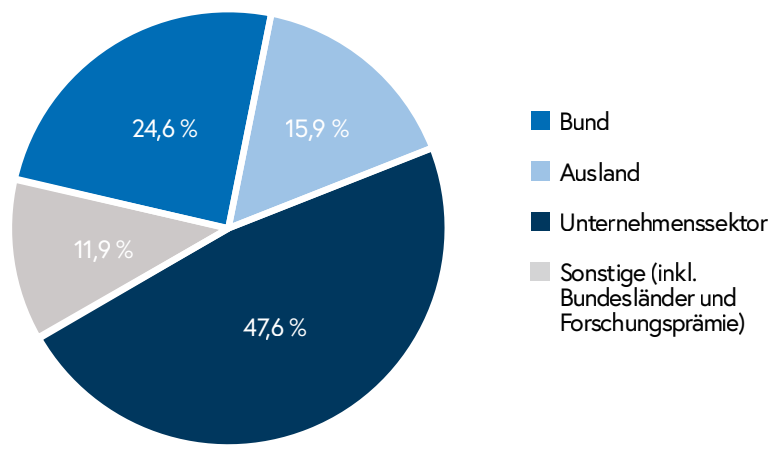

Quelle: Statistik Austria, Globalschätzung vom 28.04.2020.

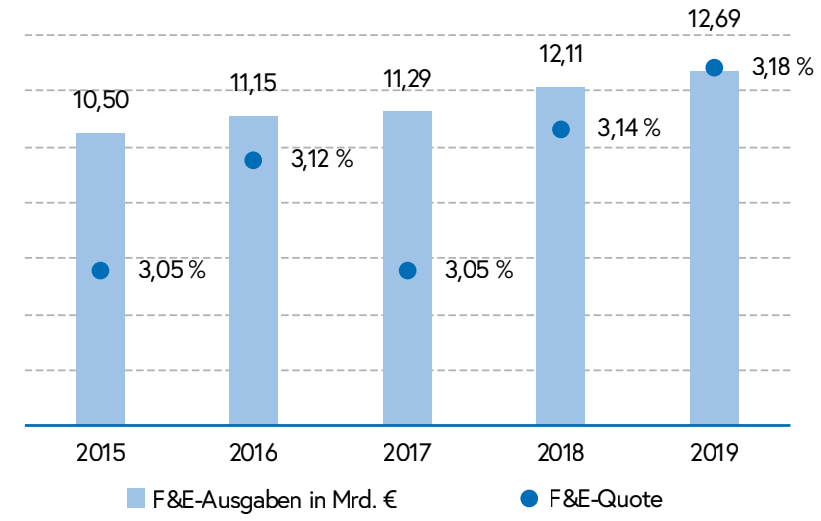

Quelle: Statistik Austria, Globalschätzung vom 28.04.2020.

Der Bund hat 2019 rund 3,12 Mrd. $€$ für F\&E ausgegeben, das entspricht etwa einem Viertel $(24,6 \%)$ der gesamten in Österreich durchgeführten F\&E. Die Bundesländer haben 2019 rund 0,55 Mrd. $€(4,3 \%)$ für $F \& E$ ausgegeben. Mit 6,04 Mrd. $€$ entfiel fast die Hälfte (47,6 \%) aller F\&E-Ausgaben auf die heimischen Unternehmen. 2,02 Mrd. $€(15,9 \%)$ wurden vom Ausland finanziert, wobei dieser Betrag zum größten Teil von ausländischen Unternehmen für in Österreich durchgeführte $F \& E$ und Rückflüsse aus den EU-Forschungsprogrammen umfasst. Auf die Forschungsprämie entfielen 2019758 Mio. $€$, was etwa $6 \%$ der F\&E-Ausgaben entspricht.

\section{Internationale Position: Österreich vielfach im vorderen Mittelfeld}

\section{F\&E-Quote $2018^{1}$}

In Bezug auf die F\&E-Ausgaben für Forschung und Entwicklung gehört Österreich zu den führenden $\mathrm{Na}-$ tionen: Mit einer F\&E-Quote von 3,17\% im Jahr 2018 weist Österreich hinter Schweden den zweithöchsten Wert innerhalb der EU-28 auf.

\footnotetext{
1 Da ein internationaler Vergleich mit rezenteren Zahlen nicht möglich ist, wird hier die F\&E-Quote 2018 verwendet. Aufgrund unterschiedlicher zeitlicher Datenverfügbarkeit unterscheiden sich die Werte für die F\&E-Quote 2018 gemäß Eurostat (3,17 \%) und der Globalschätzung von Statistik Austria (3,14 \%) leicht.
}

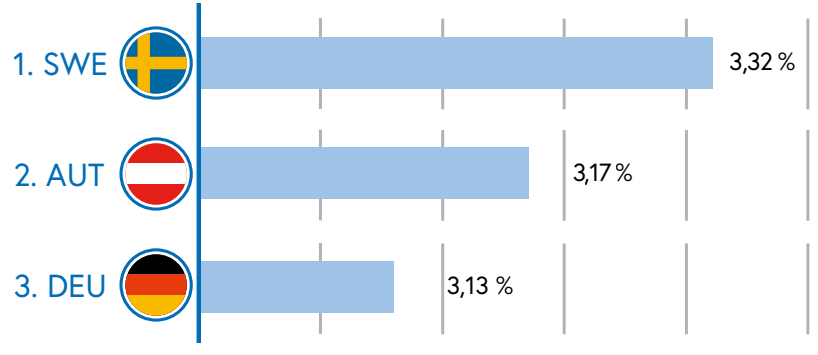

Quelle: Eurostat (2020). 
Patente pro 1.000 F\&E-Beschäftigte

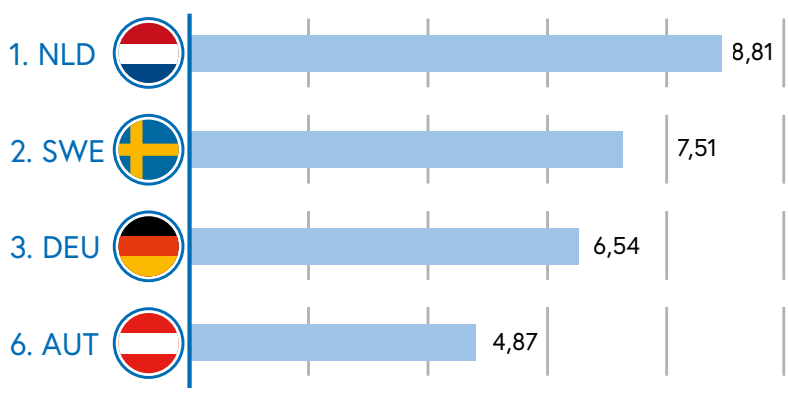

Quelle: OECD (2019).

Im Jahr 2017 liegt Österreich im EU-28-Vergleich mit 4,87 Patentanmeldungen pro 1.000 F\&E-Beschäftigte auf dem sechsten Platz. Patente spiegeln den technologischen und wirtschaftlichen Gehalt von Erfindungen wider, da Patentanmeldungen gemeinhin als Indikator für die Nutzung von Erfindungen angesehen und so Rückschlüsse auf die Innovationsfähigkeit von Ländern gezogen werden.
Wissenschaftliche Artikel pro 1.000 Einwohnerinnen und Einwohner

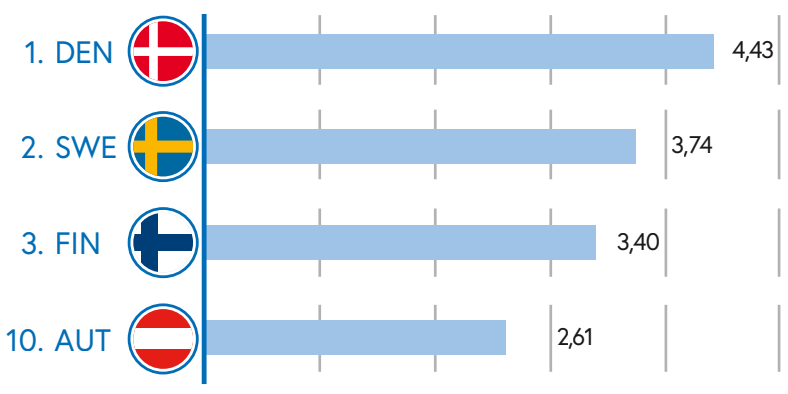

Quelle: Scimago Journal \& Country Rank (2019).

Österreich liegt mit 2,61 zitierbaren publizierten Artikeln pro 1.000 Einwohnerinnen und Einwohner auf dem 10. Platz innerhalb der EU-28 und damit im oberen Mittelfeld. Die Anzahl wissenschaftlicher Publikationen ist ein wichtiger Indikator für die wissenschaftliche Leistung eines Landes. Forschungsergebnisse sind erst dann relevant, wenn sie nach außen dokumentiert werden und zitierfähig sind.

\section{Technologische und wissenschaftliche Leistungs- und Innovationsfähigkeit}

Im globalen Vergleich liegt die technologische und wissenschaftliche Leistungs- und Innovationsfähigkeit Österreichs im guten oberen Mittelfeld. Zwar ist Österreich der Sprung in das InnovationsSpitzenfeld bisher noch nicht gelungen, aber das Land zählt zu den Strong Innovators und liegt deutlich über dem EU-Durchschnitt des European Innovation Scoreboard 2019.

Global Competitiveness
Index 2019
Rang 21 von 141

European Innovation
Scoreboard 2019
Rang 9 von 28

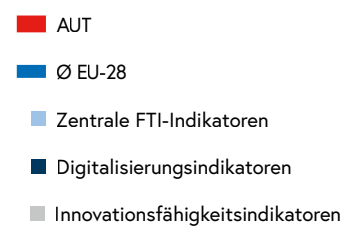

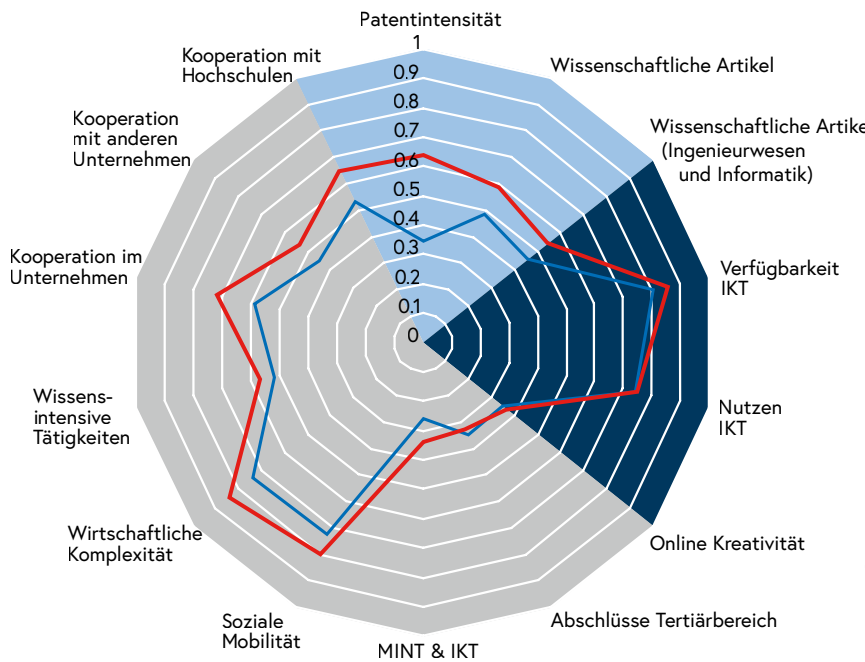

Global Innovation Index 2019

Rang 21 von 129

Innovationsindikator 2020

Rang 9 von 35

Österreich nimmt, mit Ausnahme der Tertiärabschlüsse, deren Normierung aber nicht europaweit einheitlich ist, in allen Aspekten, welche die Innovationsfähigkeit darstellen, eine im Vergleich zum EUDurchschnitt bessere Position ein. 
Index für die digitale Wirtschaft und Gesellschaft (DESI) 2019

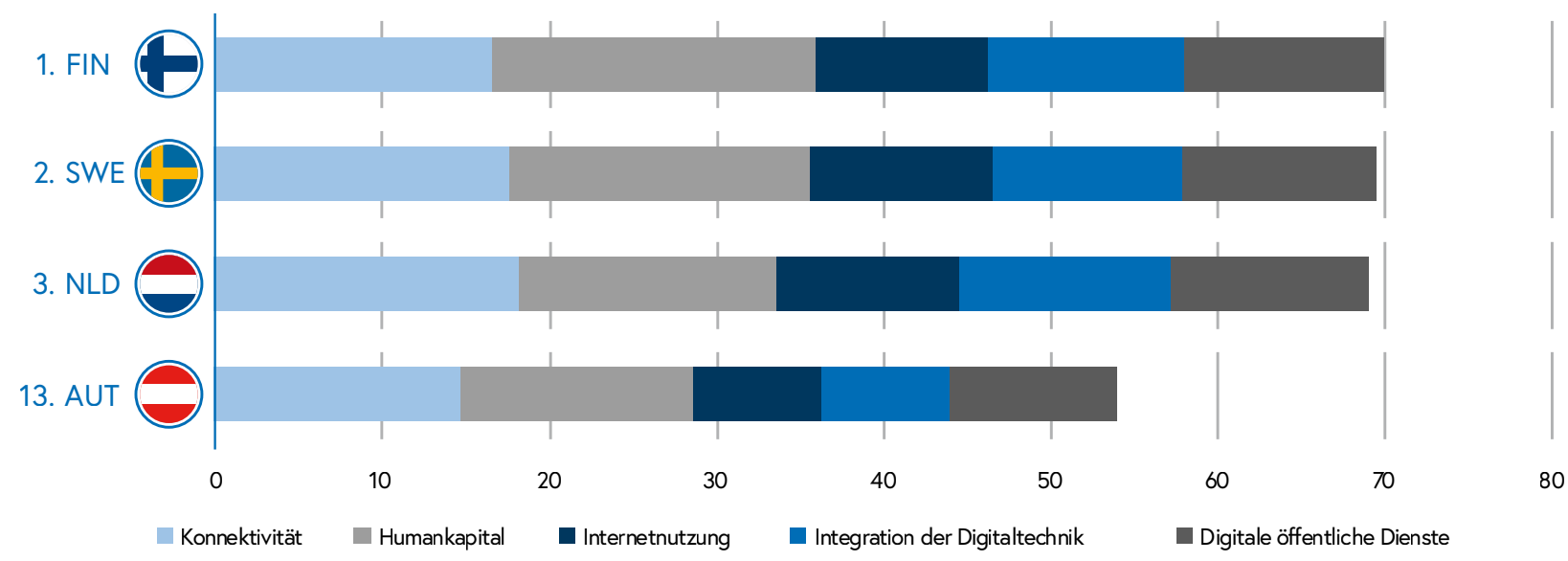

Quelle: Europäische Kommission (2019f).

Im Bereich Digitalisierung liegt Österreich insgesamt im europäischen Mittelfeld. Insbesondere die digitalen Kompetenzen der Einwohnerinnen und Einwohner stellen eine Stärke Österreichs dar. Auch die Verfügbarkeit von Informations- und Kommunikationstechnologien ist im internationalen Vergleich relativ stark ausgeprägt. Im Bereich E-Government liegt Österreich gemäß E-Government Benchmark 2019 der EU-Kommission europaweit auf dem dritten Platz hinter Malta und Estland. Handlungspotenziale liegen für Österreich im internationalen Vergleich vor allem in der Nutzung von Informations- und Kommunikationstechnologien durch private Haushalte und in der Nutzung von Big Data und Clouds durch Unternehmen.

\section{Österreich in Horizon 2020 sehr gut positioniert}

\section{Horizon 2020 Bewilligungssummen und Erfolgsquoten nach Sektoren}

Die Gesamtsumme der Bewilligungen für Österreich in Horizon 2020 liegt bei 1,46 Mrd. €. Über die gesamte Programmperiode 2014-2020 beträgt die Erfolgsquote Österreichs 18,2 \%. Österreich liegt damit hinter Belgien an zweiter Stelle der EU-28 und deutlich über dem EU-Durchschnitt.

Die österreichischen Universitäten bzw. Hochschulen konnten 497,6 Mio. $€$ einwerben, Unternehmen 465,9 Mio. $€$ und außeruniversitäre Forschungseinrichtungen 348,9 Mio. $€$. Letztere verzeichnen mit $20,0 \%$ allerdings die höchste Erfolgsquote der österreichischen Akteure. Zudem liegen österreichische Unternehmen mit einer Erfolgsquote von 18,1\% europaweit an der Spitze. Diese Werte sind das Zeugnis eines hohen Niveaus der österreichischen F\&E und sind auch ein Indiz für eine professionelle Beratungsund Betreuungsstruktur.

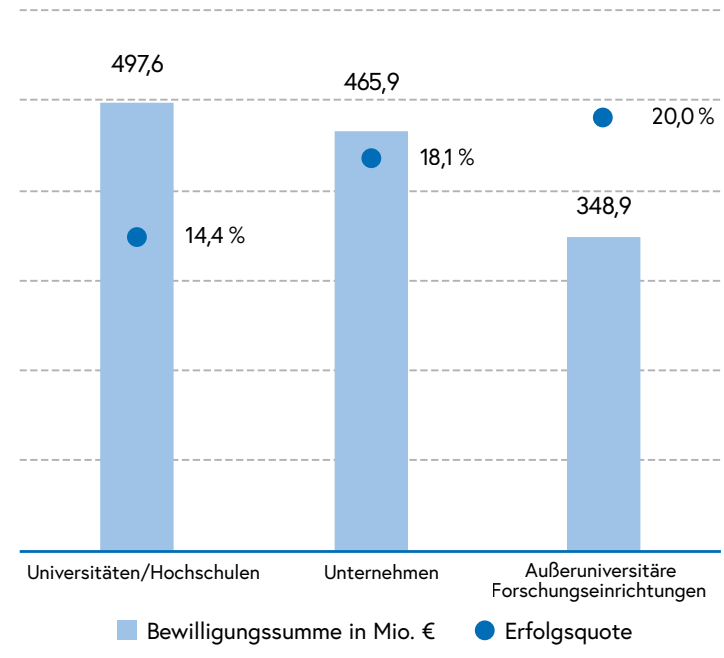

Quelle: EK/FFG per 03/2020. 


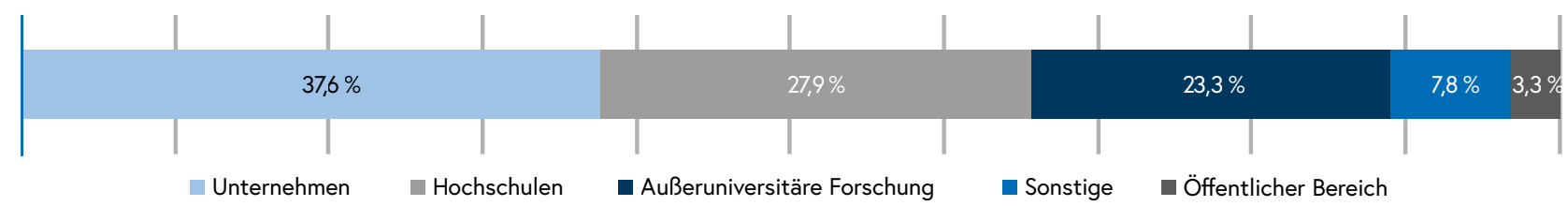

Quelle: EK/FFG per 03/2020.

Die meisten österreichischen Beteiligungen in Horizon 2020 stammen mit 37,6\% aus dem Unternehmenssektor (bezugnehmend auf die Gesamtanzahl), davon sind fast zwei Drittel kleine und mittlere Unternehmen. Danach folgen der Hochschulsektor (27,9\%) und der außeruniversitäre Forschungssektor (23,3 \%). Insgesamt machen diese drei Sektoren rd. 89 \% der österreichischen Beteiligungen in Horizon 2020 aus.

\section{Österreichische Universitäten und Unternehmen: aktiv in KI}

Unter „Künstlicher Intelligenz“ werden künstliche Systeme verstanden, die intelligentes ( $=$ selbst lernendes) Verhalten aufweisen und deshalb mit einem gewissen Grad an Autonomie agieren.

Die wesentlichsten Forschungsbeiträge im Bereich der KI werden international von Universitäten publiziert. Forschung zu KI hat in den vergangenen Jahren an den österreichischen Universitäten stark zugenommen. 18 der 22 österreichischen Universitäten haben im Zeitraum 2016-2018 Publikationen zu KI veröffentlicht oder an EU- oder FWF-Projekten mit einem Schwerpunkt auf KI teilgenommen. Österreichs Stärken liegen insbesondere in den anwendungsorientierten Forschungsfeldern Expert Systems, Robotics, Machine Learning und Autonomous Systems.

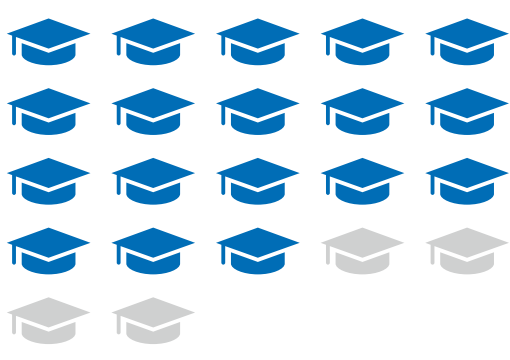

Quelle: Heller-Schuh et al. (2019).

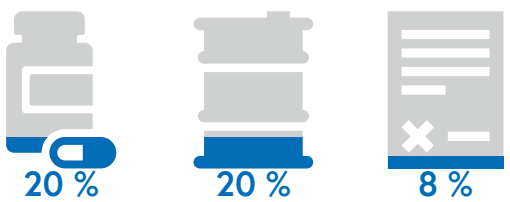

Quelle: Prem \& Ruhland (2019).
Insgesamt konnten ca. 600 Unternehmen in Österreich, die im Themenkomplex $\mathrm{KI}$ aktiv sind, identifiziert werden. Dies entspricht einem relativ kleinen Anteil aller Unternehmen. Die höchste Dichte von KI-Unternehmen findet sich im Bereich der Herstellung pharmazeutischer Erzeugnisse (20\%), der Mineralölverarbeitung (20\%) und Versicherungen (8 \%). Österreichische Unternehmen wenden KI vor allem für die Automatisierung, Prozessoptimierung und Effizienzsteigerung an.

\section{Evaluierungskultur und -praxis}

Evaluierungen fungieren als wichtiges Instrument der FTI-Politik und Governance, das dazu beiträgt, Transparenz, Rechenschaftslegung und eine evidenzbasierte Entscheidungsfindung zu unterstützen. Österreich liegt hinsichtlich der Anzahl von Evaluierungen im FTI-Bereich im europäischen Spitzenfeld. Studien, die sich dem Umgang mit Evaluierungen widmen, unterstreichen die im Allgemeinen hohe Professionalität und Qualität österreichischer Evaluierungen. Die meisten Organisationen, die Evaluierungen beauftragen und/oder durchführen, sind Mitglieder der Österreichischen Plattform für Forschung- und Technologiepolitikevaluierung (fteval) und orientieren sich an den von der Plattform formulierten Standards (https://www.fteval.at/content/home/standards/fteval_standards/). 